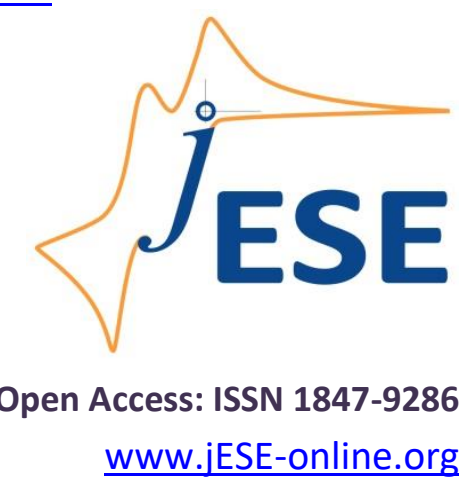

Original scientific paper

\title{
Protective films of stearic and octadecylphosphonic acid formed by spray coating
}

\author{
Ekatarina Kristan Mioč, Helena Otmačić Ćurković ${ }^{凶}$ \\ Laboratory for Corrosion Engineering and Surface Protection - ReCorr, Department of Electro- \\ chemistry, Faculty of Chemical Engineering and Technology, University of Zagreb, Marulićev trg 19, \\ 10000, Zagreb, Croatia
}

Corresponding author: ${ }^{\bowtie}$ helena.otmacic@fkit.hr; Tel.: +385-1-4597-117

Received: October 22, 2019; Revised: January 18, 2020; Accepted: January 28, 2020

\begin{abstract}
Spray coating formation of stearic and octadecylphosphonic acid films for corrosion protection of cupronickel alloy was studied in this work as a more practical alternative to widely studied dip-coating method. Protective properties of organic films formed under various experimental conditions were examined by electrochemical studies in $3 \% \mathrm{NaCl}$ solution as a corrosive medium. Polarization resistance measurements as well as electrochemical impedance spectroscopy were employed to follow in time the corrosion behaviour of cupronickel alloy modified by studied organic acids. It was found that among examined experimental parameters, time elapsed between two sprays and number of sprays have the strongest influence on the film stability and its protective properties. This study confirmed that it is possible to form by spray coating the films of stearic and octadecylphosphonic acid with protective properties that resemble to those of the films prepared by dip-coating method. Differences in corrosion behaviour of samples protected with stearic and octadecylphosphonic acid were attributed to difference in the bond strength between substrate and each organic acid. Studied samples were also examined by the scanning electron microscopy. Fourier transformed infrared spectroscopy studies showed that crystalline structure dominates in studied films, while contact angle measurements confirmed that modified cupronickel alloy surface exhibits hydrophobic properties.
\end{abstract}

\section{Keywords}

Corrosion; electrochemical impedance spectroscopy; polarization measurements, FTIR, cupronickel alloy.

\section{Introduction}

Modification of surface properties of metallic or semiconductor substrates by long-chain organic molecules has been thoroughly studied in recent years due to interest for its application in various 
fields such as organic electronics, sensors or corrosion protection [1-7]. Molecules with sufficiently long alkyl chain usually possess the self-assembling property, i.e. they spontaneously form ordered structures in a form of self-assembled monolayers or multilayers $[8,9]$. Such thin films present a barrier between the substrate and environment. If the film has a low number of defects and is strongly attached to metallic substrate it can provide good corrosion protection to a metal in various corrosive environments. It has been shown that long-chain organic acids such as carboxylic, phosphonic or hydroxamic acids, can form such barrier layers on different metals [6,7,10-15]. In most of cases, self-assembled monolayers/multilayers were prepared by dip-coating method. Although this method is characterized by good reproducibility, its drawback is that it is time consuming and requires high amounts of solution. Spraying method is often used for organic coatings as it enables their quick application on metallic objects of different dimensions. Despite advantages over dip-coating method, there are only few works where spraying method was used for formation of self-assembled monolayers $[10,11,13,16,17]$. Studies on films formed by dipcoating method showed that many different parameters should be taken into account in obtaining a layer with small number of defects and high crystallinity [15]. Among the most important parameters is the adsorption time, as a certain time is always needed for molecules to reach the metal surface, adsorb on it and organize in well-ordered structures [18]. Studies performed on nickel oxide modification by alkylphosphonic acids self-assembled monolayers also showed that wellordered monolayers can be formed by both immersion and aerosol spraying methods, if adequate conditions for monolayer formation were selected [11]. In our previous studies it was shown that multilayer films of stearic acid (SA) [14,15] or octadecylphosphonic acid (ODPA) [7] formed by immersion method provide high and durable corrosion protection to copper-nickel alloy (CuNI) in chloride medium. The aim of this work is to determine under which conditions SA films that provide good corrosion protection to underlying CuNi substrate, can be formed by spraying method. Then, similar experimental conditions were applied for preparation of ODPA films.

\section{Experimental}

\section{Sample preparation}

Investigations were performed on cupronickel alloy (70Cu30Ni) obtained from Goodfellow Inc., UK. The specimens were cut-out from a cupronickel rod with $1.3 \mathrm{~cm}$ diameter and $0.5 \mathrm{~cm}$ in thickness. In order to prepare working electrodes for electrochemical measurements on the backside of these plates, a copper wire was soldered and then, the electrodes were embedded into epoxy resin. The exposed surface area of the working electrodes was $1.33 \mathrm{~cm}^{2}$. Prior to all investigations and surface modifications, the electrodes were abraded with emery paper grade 800, 1200, 2500, and polished with $\alpha-\mathrm{Al}_{2} \mathrm{O}_{3}$ particle size $0.1 \mu \mathrm{m}$, degreased with ethanol in ultrasonic bath and rinsed with re-distilled water.

Stearic acid and octadecylphosphonic acid (ODPA, $97 \%$ ) were obtained from Sigma-Aldrich Corp. USA, while sodium chloride (p.a.) and ethanol (96\% p.a.) were obtained from Lachner d.o.o. Croatia. Solutions for electrochemical measurements were prepared with deionized water. Ethanolic solutions of $0.01 \mathrm{~mol} \mathrm{dm}^{-3}$ stearic and octadecylphosphonic acid were used for spray application. The first step was to optimize the method of preparing the films with stearic acid, and after specific preparation procedure was chosen, it was applied for preparation of ODPA films. The film formation was conducted according to the experimental procedures presented in Table 1. They consisted of three steps: substrate oxidation which was conducted in order to obtain reproducible oxide layer for $24 \mathrm{~h}$ at $80^{\circ} \mathrm{C}$, followed by acid adsorption from EtOH solution of SA or ODPA by spraying method, 
and final drying step, $5 \mathrm{~h}$ at $50^{\circ} \mathrm{C}$ (SA) or $80^{\circ} \mathrm{C}$ (ODPA) [7,14]. Initially, all the samples were sprayed 5 times while the influence of temperature $(A)$ and time $(B)$ between two consecutive spraying on the protective properties of SA film were studied. In the next step, the influence of the number of sprays $(C)$ on the protective properties of the formed film was examined. For comparison, a blank sample (CuNi) was prepared, on which native oxide layer was formed during 24 hours at $80{ }^{\circ} \mathrm{C}$. The oxidation and drying were conducted in an air convection oven with temperature control accuracy of $0.5^{\circ} \mathrm{C}$.

Table 1. Investigated film preparation procedures by spraying method

\begin{tabular}{ccccc}
\hline & Sample & Temperature, ${ }^{\circ} \mathrm{C}$ & Time, min & Number of sprays \\
\hline A) Influence of substrate & $\mathrm{T} 25$ & $\mathbf{2 5}$ & 60 & 5 \\
temperature & $\mathrm{T} 40$ & $\mathbf{4 0}$ & 60 & 5 \\
\hline \multirow{2}{*}{ B) Influence of time between } & $\mathrm{t} 10$ & 25 & $\mathbf{1 0}$ & 5 \\
spraying & $\mathrm{t} 30$ & 25 & $\mathbf{3 0}$ & 5 \\
& $\mathrm{t} 60$ & 25 & $\mathbf{6 0}$ & 5 \\
\hline \multirow{2}{*}{ C) Influence of the number of } & $2 \mathrm{x}$ & 25 & 30 & $\mathbf{2}$ \\
sprays & $5 \mathrm{x}$ & 25 & 30 & $\mathbf{5}$ \\
& $10 \mathrm{x}$ & 25 & 30 & $\mathbf{1 0}$ \\
\hline
\end{tabular}

\section{Electrochemical measurements}

Electrochemical investigations were conducted in a three electrode cell $\left(250 \mathrm{~cm}^{3}\right)$ containing $3 \%$ $\mathrm{NaCl}$ solution. A platinum foil and saturated calomel electrode were used as the counter and reference electrode, respectively. Studies were conducted at room temperature, $24 \pm 2{ }^{\circ} \mathrm{C}$. The polarization measurements were performed in a wide $( \pm 150 \mathrm{mV}$ vs. open circuit potential) and narrow ( $\pm 20 \mathrm{mV}$ $v s$. open circuit potential) potential range, at a potential scan rate of $0.166 \mathrm{mV} \mathrm{s}^{-1}$. The electrochemical impedance spectroscopy (EIS) was performed at open circuit potential $\left(E_{\mathrm{ocp}}\right)$ in the frequency range $100 \mathrm{kHz}$ to $10 \mathrm{mHz}, 5$ points per decade, and $10 \mathrm{mV}_{\mathrm{rms}} \mathrm{AC}$ amplitude. The obtained impedance spectra were interpreted on the basis of electrical equivalent circuits using ZSimpWin software. In general, $\chi^{2}$ value was always below $5 \cdot 10^{-4}$. The electrochemical measurements were performed using a Bio-Logic SP-300 potentiostat. All measurements were conducted in triplicate.

\section{Surface studies}

Contact angle (CA) measurements, Fourier transform infrared spectroscopy (FTIR) and scanning electron microscopy (SEM) were performed with the aim of surface layer characterization. The contact angle measurements on bare cupronickel and SA and ODPA treated CuNi samples were conducted using a goniometer DataPhysics Contact Angle System OCA 20, with a drop of $2 \mu \mathrm{L}$ water under the ambient atmospheric conditions. All measurements were conducted in at least ten points. SEM morphology analysis was performed with VEGA 3 SEM TESCAN at an acceleration voltage of $10 \mathrm{kV}$. FTIR measurements were carried out by attenuated total reflectance Fourier transform infrared spectroscopy (ATR FTIR), using a Spectrum One FTIR spectrometer from Perkin Elmer, with the scan range from $4000-650 \mathrm{~cm}^{-1}$, having a resolution of $0.5 \mathrm{~cm}^{-1}$, and the results shown in this paper were averages of 25 scans.

\section{Results and discussion}

In order to determine the experimental conditions at which SA film with excellent barrier properties is formed, protective properties of differently formed samples were tested by polarization measurements in $3 \% \mathrm{NaCl}$ solution. 


\section{Polarization measurements of SA treated samples}

Influence of temperature between spraying

Since our previous studies on samples prepared by immersion method showed that adsorption of SA and ODPA is enhanced at elevated temperature $[7,14,15]$, there was a need to investigate the influence of temperature on the adsorption step in the spraying method. According to the literature [19], lower activation energy is required for a molecular gas or liquid precursor dispersed in small droplets at room temperature to bind by the chemisorption on a heated surface of the substrate, than for the heated solution and cold substrate. Accordingly, it is expected that heating of the substrate during spraying could improve adsorption of molecules on the substrate. Moreover, by spraying droplets of a room temperature in comparison to that of elevated temperature, the solvent evaporation during spraying is reduced. The solvent evaporation changes the droplet size, which affects the concentration and spatial arrangement of a drop, resulting in uneven spraying of droplets and disordered layer on the substrate surface $[20,21]$. For above mentioned reasons, SA solution was not heated as in the case of immersion method $[7,14]$. Instead of that, between two consecutive sprays, T40 sample was heated at $40^{\circ} \mathrm{C}$ for $1 \mathrm{~h}$, after which it was sprayed with a solution of room temperature. The sample thus prepared was compared to T25 sample that was left at room temperature.

The protective properties of such prepared samples were tested by polarization in a wider range of potentials after $1 \mathrm{~h}$ of exposure to corrosive solution (Figure 1a). Polarization measurements were also conducted in a narrow potential range during 14 days of exposure of samples to corrosive medium. From such obtained polarization curves, polarization resistance values were calculated (Figure 1b).

(a)

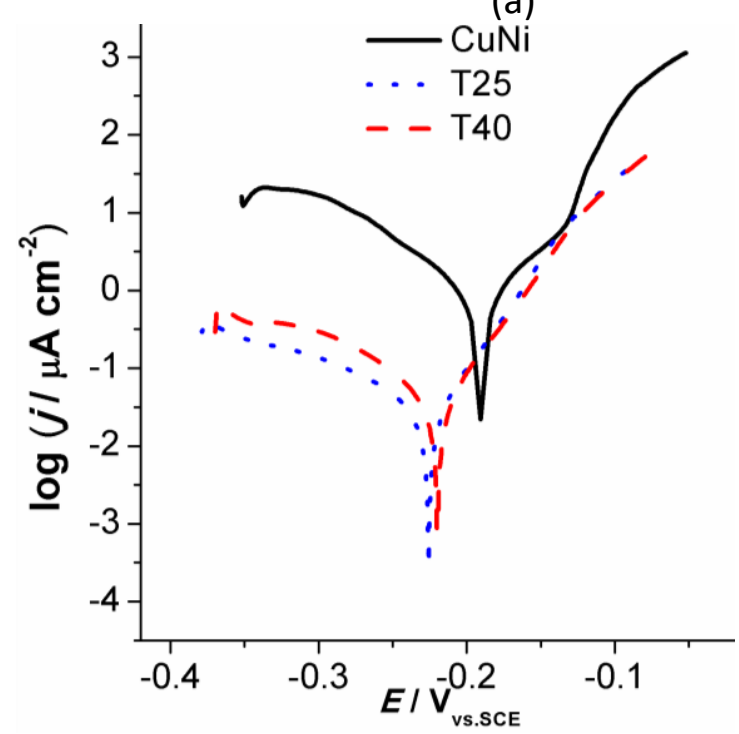

(b)

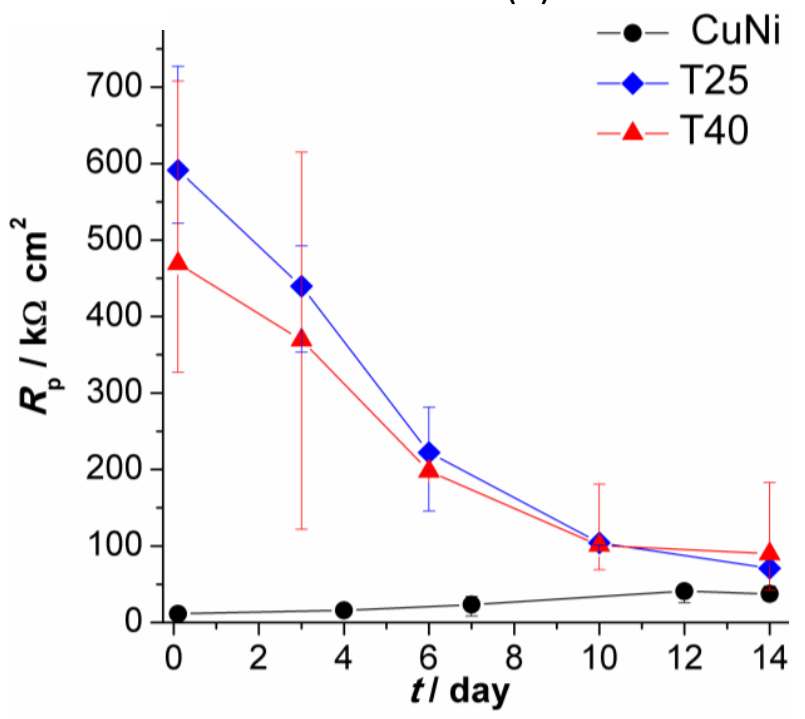

Figure 1. Influence of temperature between spraying: (a) polarization curves recorded after one-hour immersion in $3 \% \mathrm{NaCl}$ and (b) polarization resistance as a function of immersion time for blank and treated samples

Table 2 presents corrosion parameters, i.e. corrosion potential $\left(E_{\text {corr }}\right)$, corrosion current density $\left(j_{\text {corr }}\right)$, anodic and cathodic Tafel slopes $\left(b_{a}\right.$ and $\left.b_{c}\right)$, obtained from the polarization curves (Figure 1a) by Tafel extrapolation method. From the values of corrosion current densities of blank $\left(j_{\text {corr }}{ }^{0}\right)$ and treated $\left(j_{\text {corr }}{ }^{t}\right)$ samples, corrosion inhibition efficiency $\left(\eta_{\mathrm{i}}\right)$ was calculated according to the following equation:

$$
\eta_{\mathrm{i}} / \%=\frac{j_{\text {corr }}{ }^{0}-\mathrm{j}_{\text {corr }}^{\mathrm{t}}}{j_{\text {corr }}{ }^{0}} 100
$$


Table 2. Corrosion parameters obtained by Tafel extrapolation method from polarization curves given in Fig. 1a Values in parentheses represent standard deviations

\begin{tabular}{cccccc}
\hline Sample & $E_{\text {corr }} / \mathrm{mV}_{\text {vs. SCE }}$ & $j_{\text {corr }} / \mu \mathrm{Acm}^{-2}$ & $b_{\mathrm{a}} / \mathrm{mV} \mathrm{dec}^{-1}$ & $-b_{\mathrm{c}} / \mathrm{mV} \mathrm{dec}^{-1}$ & $\eta_{\mathrm{i}} / \%$ \\
\hline $\mathrm{CuNi}$ & $-177(6)$ & $1.12(0.10)$ & $78(21)$ & $95(5)$ & - \\
$\mathrm{T} 25$ & $-232(6)$ & $0.03(0)$ & $43(3)$ & $109(5)$ & $97(0)$ \\
$\mathrm{T} 40$ & $-232(9)$ & $0.06(0.01)$ & $46(3)$ & $124(22)$ & $94(1)$ \\
\hline
\end{tabular}

From the polarization curves presented in Figure 1a and corrosion parameters given in Table 2, it is clear that corrosion current density of both SA treated samples is significantly lower compared to that of the blank sample. The protective films on T25 and T40 samples acted as barrier toward oxygen diffusion, thus slowing down cathodic corrosion reaction, while anodic corrosion reaction decreased to a lesser extent. This effect was observed in most of papers investigating use of selfassembled monolayers (SAMs) in corrosion protection [22,23].

For application of SAMs in corrosion protection, it is not sufficient to determine only the initial protection level but also to verify if the protection remains satisfactory with passing of time. For that reason, polarization DC measurements in narrow potential range were conducted over longer period. The polarization resistance $\left(R_{\mathrm{p}}\right)$ values determined from polarization measurements are given in Figure 1b. It can be seen that both T25 and T40 samples initially exhibited higher $R_{\mathrm{p}}$ values compared to the untreated sample, but over time there was a decrease in these values. Decrease in polarization resistance in time can be associated with dissolution of upper layers of the protective SA film, or formation of pores in the film. However, the minimum $R_{\mathrm{p}}$ values observed after 14 days of immersion in $3 \% \mathrm{NaCl}$ solution are twice than of the untreated sample. The T25 sample showed the highest initial polarization resistance values with higher reproducibility than for T40 sample. Such results can be related to slower solvent evaporation at lower temperatures which then enable longer time for organization of SA molecules on CuNi substrate, i.e. molecular motion and stacking into a more compact film. Study of spread coating of ODPA on mica showed that for longer time of adsorption (before solvent evaporation), less porous and more ordered film was formed [24]. This was found when deposition was conducted by low concentration ODPA solution containing dissolved single molecules, and also in the case of more concentrated solution containing ODPA micelles. Therefore, even though heating of the substrate during spraying can improve adsorption of molecules on a substrate [19], faster evaporation of the solvent can lead to poorer organization of molecules in the layer and consequently to formation of less protective ODPA film.

\section{Influence of time between spraying}

After examining the substrate temperature effect, the next parameter tested was the time elapsed between two consecutive sprays. For that purpose, samples were sprayed at room temperature five times, but between two consecutive sprays samples were left in air for 60 minutes ( $\mathrm{t} 60$ sample), 30 minutes ( $\mathrm{t} 30$ sample), or 10 minutes (t10 sample). In this way, it was attempted to determine the minimum time required for the preparation of stable and protective layers. Their protective efficiency was examined by polarization measurements as presented in Figure 2, while corrosion parameters obtained from polarization curves are presented in Table 3.

From Figure $2 a$ it can be seen that polarization curves obtained for all SA treated samples exhibit lower values of current density compared to the untreated sample, with a significant reduction of cathodic current density resulting in the displacement of the corrosion potential in the cathodic direction. 
(a)

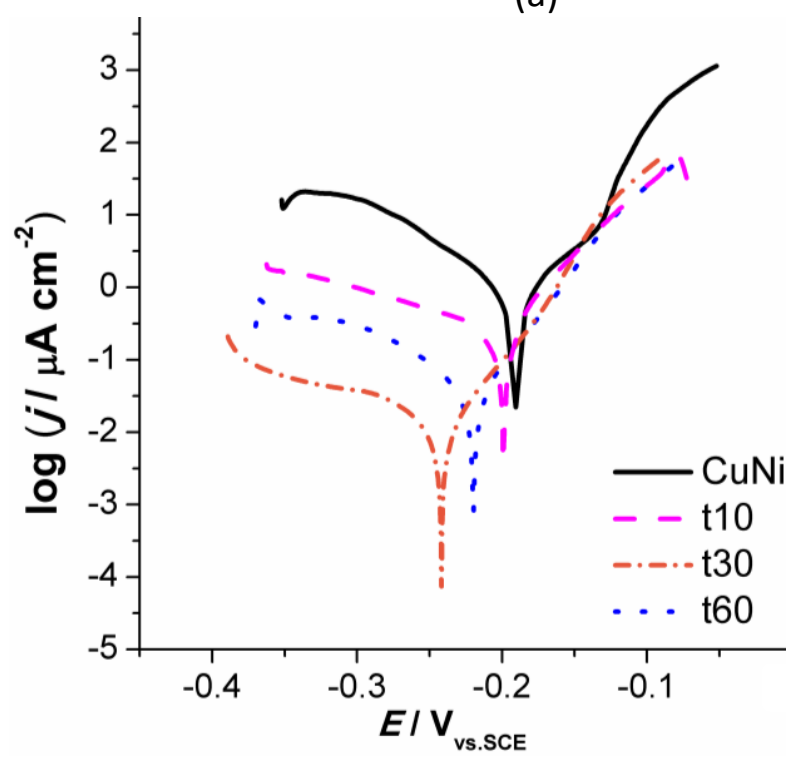

(b)

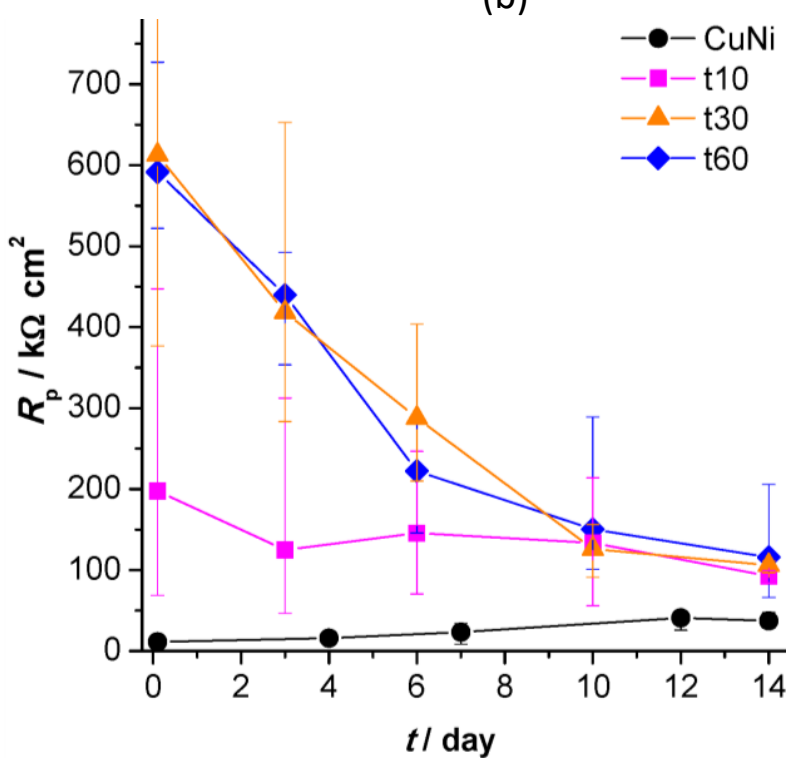

Figure 2. Influence of time between spraying - results of polarization measurements:

(a) polarization curves recorded after one hour immersion in $3 \% \mathrm{NaCl}$, and (b) polarization resistance as a function of immersion time for blank and treated samples.

Table 3. Corrosion parameters obtained by Tafel extrapolation method from polarization curves given in Fig. $2 a$ Values in parentheses represent standard deviations

\begin{tabular}{cccccc}
\hline Sample & $E_{\text {corr }} / \mathrm{mV}_{\text {vs. SCE }}$ & $j_{\text {corr }} / \mu \mathrm{A} \mathrm{cm} \mathrm{cm}^{-2}$ & $b_{\mathrm{a}} / \mathrm{mV} \mathrm{dec}^{-1}$ & $-b_{\mathrm{c}} / \mathrm{mV} \mathrm{dec}^{-1}$ & $\eta_{\mathrm{i}} / \%$ \\
\hline $\mathrm{t} 10$ & $-203(5)$ & $0.28(0.02)$ & $37(9)$ & $147(23)$ & $75(2)$ \\
$\mathrm{t} 30$ & $-234(12)$ & $0.02(0.01)$ & $39(2)$ & $89(7)$ & $98(1)$ \\
$\mathrm{t} 60$ & $-232(6)$ & $0.03(0)$ & $43(3)$ & $109(5)$ & $97(0)$ \\
\hline
\end{tabular}

The largest decrease can be observed for $\mathrm{t} 30$ sample which indicates formation of an effective barrier to oxygen diffusion towards the metal surface, probably due to the formation of a layer with less defects and pores. From corrosion parameters shown in Table 3, it can also be noted that the lowest value of corrosion current density and hence the lowest corrosion rate and the highest corrosion inhibition efficiency was obtained for $\mathrm{t} 30$ sample. Similar results were obtained by testing the durability of protection by polarization resistance measurements in time, as can be seen in Figure $2 \mathrm{~b}$. From the obtained results it can be seen that 10 minutes was not sufficient time between two sprays, while time longer than 30 minutes was even not required, as it didn't contribute to improvement of protective properties of SA film.

Influence of the number of sprays

The next step in optimizing the spraying method was to examine how many sprays are needed to obtain good corrosion protection. As aforementioned, it is expected that thicker SA layers would provide better corrosion protection. However, if such layers are porous and with high number of defects, their protective effect is not very high. Therefore, our aim was to prepare sufficiently thick protective layer, but with low degree of porosity in order to achieve good corrosion protection. Therefore, the prepared samples were sprayed at room temperature two ( $2 x$ sample), five ( $5 x$ sample) and ten times (10x sample). Between the individual spraying, samples were left for 30 minutes at room temperature. The results of the polarization measurements are shown in Figure 3 , and the parameters obtained by Tafel extrapolation method from polarization curves are shown in Table 4. 
(a)

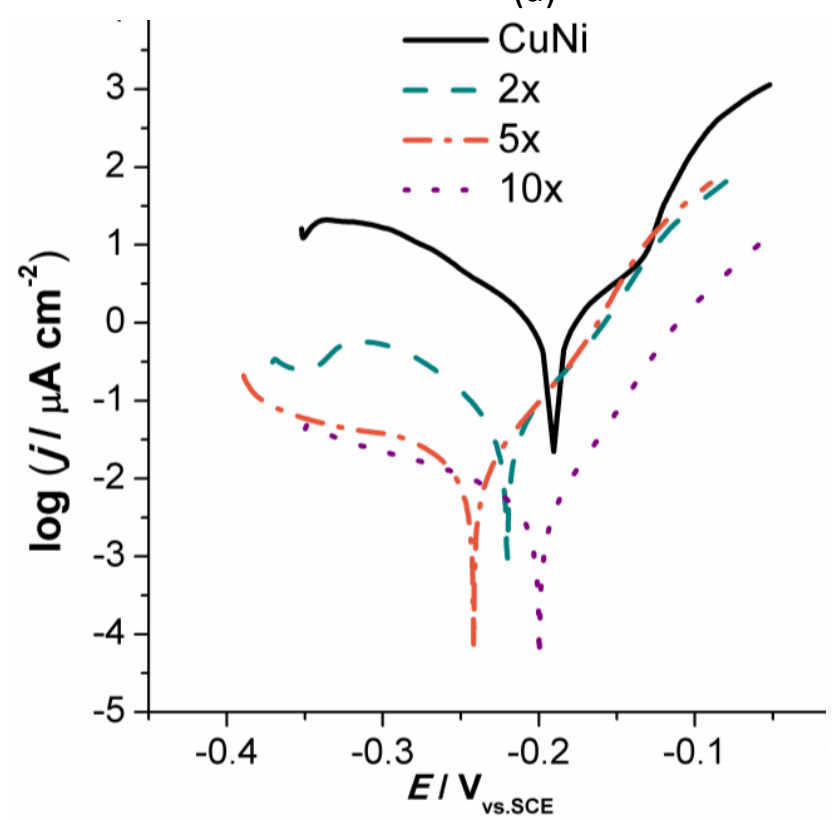

(b)

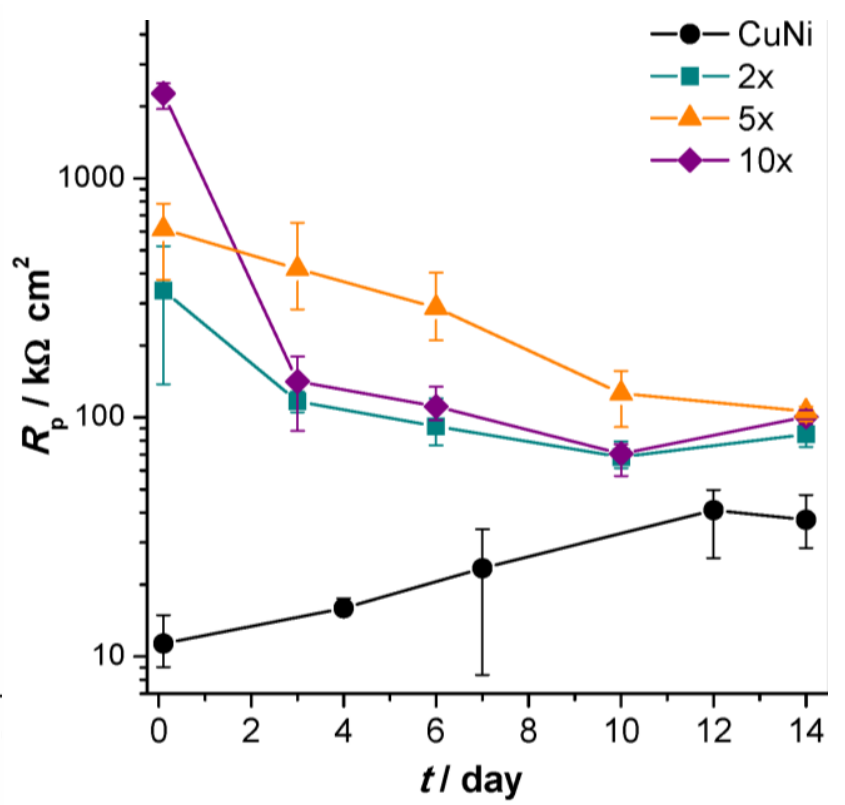

Figure 3. Influence of the number of spraying - results of polarization measurements:

(a) polarization curves recorded after one hour of immersion in $3 \% \mathrm{NaCl}$, and

(b) polarization resistances as a function of immersion time for blank and treated samples

Table 4. Corrosion parameters obtained by Tafel extrapolation method from polarization curves given in Fig. 3 Values in parentheses represent standard deviations

\begin{tabular}{cccccc}
\hline Sample & $E_{\text {corr }} / \mathrm{mV}_{\text {vs. SCE }}$ & $j_{\text {corr }} / \mu \mathrm{Acm}^{-2}$ & $b_{\mathrm{a}} / \mathrm{mV} \mathrm{dec}^{-1}$ & $-b_{\mathrm{c}} / \mathrm{mV} \mathrm{dec}^{-1}$ & $\eta_{\mathrm{i}} / \%$ \\
\hline $2 \mathrm{x}$ & $-232(10)$ & $0.23(0.03)$ & $48(8)$ & $124(61)$ & $80(2)$ \\
$5 \mathrm{x}$ & $-234(12)$ & $0.02(0.01)$ & $39(2)$ & $89(7)$ & $98(1)$ \\
$10 \mathrm{x}$ & $-225(22)$ & $0.01(0.01)$ & $42(2)$ & $184(29)$ & $98(1)$ \\
\hline
\end{tabular}

Figure 3a shows that the cathodic current density was reduced for all treated samples, while the slightest reduction could be observed for $2 x$ sample, which is in accordance with the fact that by increasing the number of sprays, a larger amount of SA is deposited on the surface thus resulting in a thicker protective layer. It is interesting to note that 10x sample showed the greatest anodic inhibition, probably because thicker multilayer structure caused more difficult penetration of water and chloride ions towards the substrate surface. From the values of corrosion parameters (Table 4), decrease of corrosion current density with an increase of the number of sprays can be observed. From the dependence of the polarization resistance on corrosive media exposure time shown in Figure $3 b$, it can be seen that although 10x sample initially shows the best results, on the third day of immersion, its $R_{\mathrm{p}}$ value is already equated with $2 \mathrm{x}$ sample. The possible reason of such result is that at the beginning, a very thick layer on 10x sample was formed due to the large number of sprays, but as the molecules in the multilayer structure were not tightly bounded, the outer layers were removed with time. Therefore, it can be concluded that the best results were achieved when the samples were sprayed $5 x$.

\section{Electrochemical impedance spectroscopy measurements on SA treated samples}

In order to better understand time evolution of polarization resistance values, EIS measurements were conducted. Figure 4 presents EIS spectra of studied samples for the first (Figure $4 \mathrm{a}$ and $4 \mathrm{~b}$ ) and $14^{\text {th }}$ day (Figure $4 \mathrm{c}$ and $4 \mathrm{~d}$ ) of exposure to $3 \% \mathrm{NaCl}$ solution. 
(a)

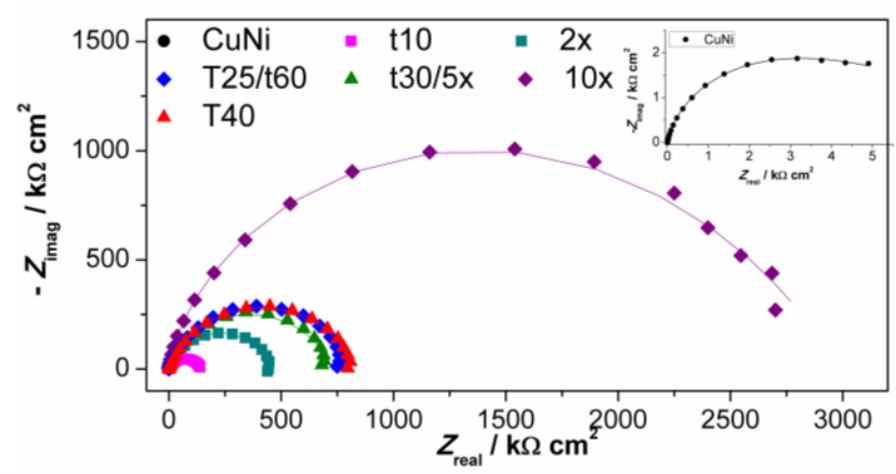

(c)

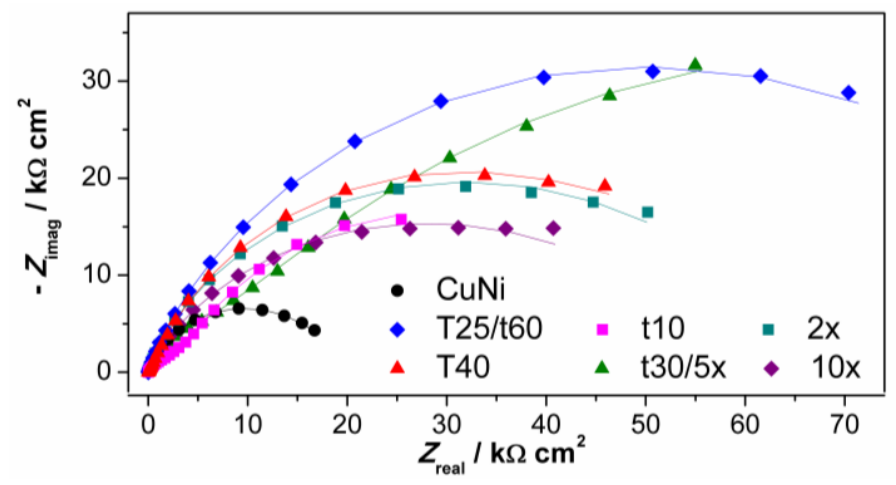

(b)

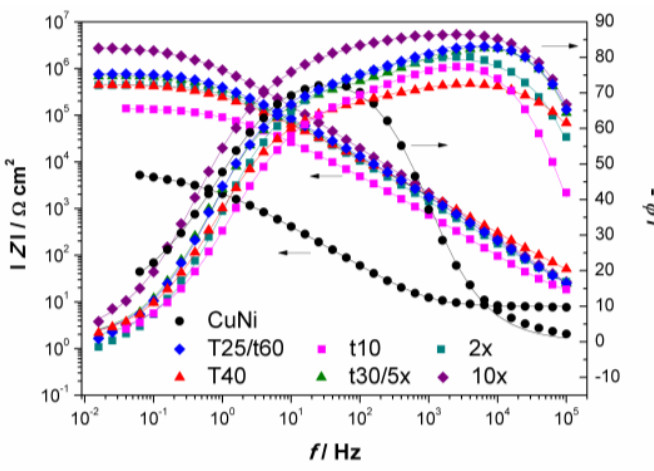

(d)

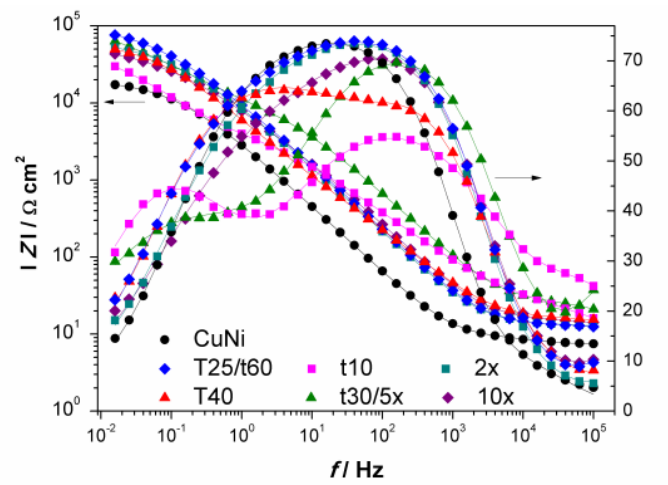

Figure 4. EIS - Nyquist and Bode plots: (a) and (b) after one hour, and (c) and (d) $14^{\text {th }}$ day of exposure to $3 \% \mathrm{NaCl}$. Symbols - experimental data; solid lines - modelled data according to electrical equivalent circuits given in Fig. 5

(a)

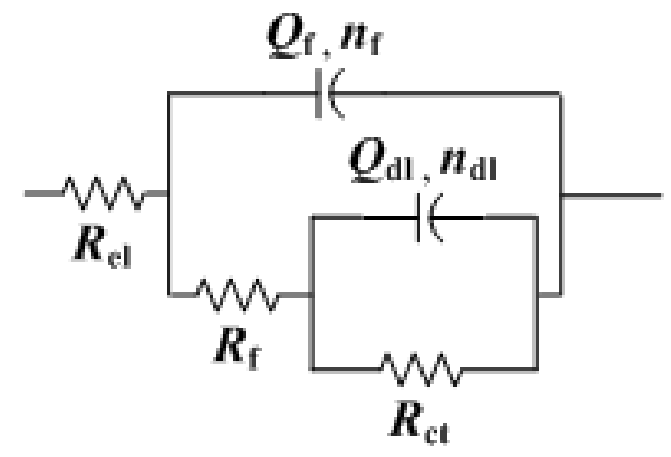

(b)

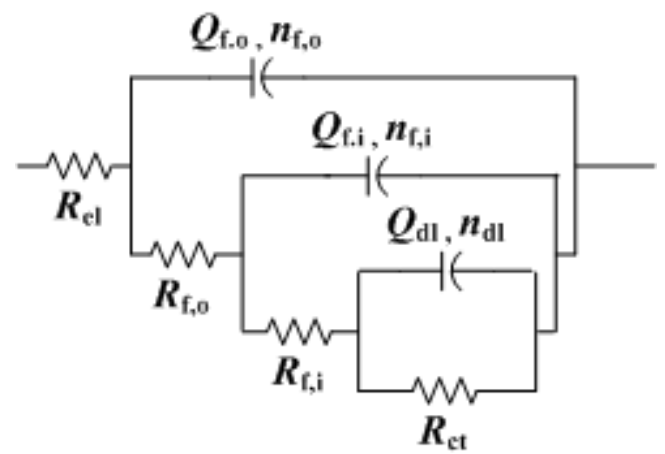

Figure 5. Equivalent electrical circuit used for fitting the impedance data

For fitting impedance data of the blank sample it was necessary to use the electrical equivalent circuit with two time constants (Figure 5a). Due to the frequency dispersion, the capacitor element in electrical equivalent circuit was replaced with the constant phase element (CPE). The impedance of $C P E\left(Z_{C P E}\right)$ is defined as

$$
Z_{\mathrm{CPE}}=1 /\left[Q(j \omega)^{n}\right]
$$

where constant $Q$ is the frequency-independent parameter of CPE [25] which represents pure capacitance when $n=1$. Proposed 2RQ circuit (Figure 5a) used for fitting EIS spectra consists of $R_{\mathrm{f}}-Q_{\mathrm{f}}$ and $R_{\mathrm{ct}}-Q_{\mathrm{dl}}$ couples. $R_{\mathrm{f}}-Q_{\mathrm{f}}$ couple describes a time constant observed at high frequencies, where $R_{\mathrm{f}}$ corresponds to the resistance of the oxide film and $Q_{\mathrm{f}}$ is related to the capacitance of the film associated with its thickness and dielectric property. $R_{\mathrm{ct}}-Q_{\mathrm{dl}}$ couple presents the corrosion reaction at the metal substrate/solution interface, where $R_{\mathrm{ct}}$ is charge transfer resistance and $Q_{\mathrm{dl}}$ is related to 
the double layer capacitance. The electrolyte resistance between the working and reference electrodes is represented by $R_{\mathrm{el}}$. For EIS spectra of SA treated samples measured on the first day of exposure to $3 \% \mathrm{NaCl}$, the same model (Figure $5 \mathrm{a}$ ) was used. In this case, however, $R_{\mathrm{f}}$ represented resistance of the pores in stearic acid film and $Q_{\mathrm{f}}$ was related to the capacitance of the surface film.

After 14 days of exposure to corrosive medium ( $3 \% \mathrm{NaCl}$ ), some changes in EIS spectra were observed (Figure $4 c$ and $4 d$ ). Firstly, the difference between impedance modulus of blank and protected samples was not as high as at the beginning of the experiment. Secondly, the third maximum in the phase angle plot appeared at high frequencies. In our previous work on stearic acid films [14], appearance of this time constant was attributed to transformation of the organic film into porous outer layer and more compact inner layer. For that reason, impedance spectra were modelled with the equivalent circuit presented in Figure $5 \mathrm{~b}$, where $R_{\mathrm{f}, \mathrm{o}}-Q_{\mathrm{f}, \mathrm{o}}$ represents resistance of the pores and capacitance of the outer porous film, and $R_{\mathrm{f}, \mathrm{i}}-Q_{\mathrm{f}, \mathrm{i}}$ represents resistance and capacitance of the inner compact layer. EIS parameters obtained by fitting selected models to experimental data are presented in Tables 5 and 6 . Note that the values for outer film parameters are omitted in Table 6 , because $R_{\mathrm{f}, \mathrm{o}}$ values were low for all studied samples such that their contribution to overall corrosion resistance is practically negligible.

Table 5. Impedance parameters for the first day of exposure to $3 \% \mathrm{NaCl}$ Values in parentheses represent standard deviations

\begin{tabular}{ccccccc}
\hline Sample & $Q_{\mathrm{f}} / \mu \mathrm{S} \mathrm{s}^{\mathrm{n}} \mathrm{cm}^{-2}$ & $n_{\mathrm{f}}$ & $R_{\mathrm{f}} / \mathrm{k} \Omega \mathrm{cm}^{2}$ & $Q_{\mathrm{dl}} / \mu \mathrm{S} \mathrm{s}^{\mathrm{n}} \mathrm{cm}^{-2}$ & $n_{\mathrm{dl}}$ & $R_{\mathrm{ct}} / \mathrm{k} \Omega \mathrm{cm}^{2}$ \\
\hline CuNi & $60.71(11.22)$ & $0.86(0.01)$ & $4.16(1.63)$ & $291.70(8.06)$ & $0.50(0)$ & $5.55(0.50)$ \\
$\mathrm{T} 40$ & $0.21(0.16)$ & $0.90(0.07)$ & $29.15(17.11)$ & $0.51(0.33)$ & $0.65(0.04)$ & $679.70(498.34)$ \\
$\mathrm{T} 25 / \mathrm{t} 60$ & $0.15(0.04)$ & $0.94(0.01)$ & $21.72(8.51)$ & $0.89(0.80)$ & $0.64(0.06)$ & $657.73(111.58)$ \\
$\mathrm{t} 30 / 5 \mathrm{x}$ & $0.13(0)$ & $0.95(0)$ & $34.87(22.78)$ & $0.26(0.13)$ & $0.65(0.05)$ & $943.73(234.92)$ \\
$\mathrm{t} 10$ & $0.42(0.18)$ & $0.90(0.01)$ & $24.28(23.48)$ & $1.11(0.59)$ & $0.63(0.07)$ & $247.67(184.95)$ \\
2x & $0.49(0.48)$ & $0.89(0.04)$ & $72.82(24.18)$ & $1.12(1.42)$ & $0.69(0.02)$ & $386.61(305.09)$ \\
10x & $0.11(0.02)$ & $0.97(0)$ & $101.73(13.97)$ & $0.12(0.03)$ & $0.67(0.08)$ & $2471.33(439.67)$ \\
\hline
\end{tabular}

By examining data in Table 5, it can be observed that the difference in impedance parameters of T40 and T25 is not significant. On the contrary, parameters of samples prepared with different times elapsed between two sprays are quite different. Firstly, t10 samples showed higher $Q_{f}$ values than t30 and $t 60$ samples. Taking in account that all samples were prepared by five times (5x) spraying, it is expected that the similar amount of SA was deposited on the metal surface and as $Q_{\mathrm{f}}$ is reversely proportional to film thickness, its values should be the same for these three samples. As this is not the case, the probable explanation is that SA layer on t10 sample was more porous, which enabled penetration of higher amounts of water into the film and resulted in increase of its dielectric constant. This is also in accordance with higher $Q_{\mathrm{dl}}$ and lower $R_{\mathrm{ct}}$ values obtained for 110 compared to t30 and t60 samples. When examining the number of sprays, it is evident that the highest resistance values (both $R_{\mathrm{f}}$ and $R_{\mathrm{ct}}$ ) and the lowest capacitance values ( $Q_{\mathrm{f}}$ and $Q_{\mathrm{dl}}$ ) were obtained for the sample with the highest number of sprays, 10x.

From data presented in Table 6, it can be observed that two weeks (14 days) exposure of samples to $3 \% \mathrm{NaCl}$ solution resulted in decrease of almost all resistive elements values and increase of capacitive elements values when compared to data in Table 5 for the first day of exposure. Such changes can be attributed to ingress of water into the SA film and dissolution of outer film layers. 
Table 6. Impedance parameters for the $14^{\text {th }}$ day of exposure to $3 \% \mathrm{NaCl}$ Values in parentheses represent standard deviations

\begin{tabular}{ccccccc}
\hline Sample & $Q_{\mathrm{f}, \mathrm{i}} / \mu \mathrm{S} \mathrm{s}^{\mathrm{n}} \mathrm{cm}^{-2}$ & $n_{\mathrm{f}, \mathrm{i}}$ & $R_{\mathrm{f}, \mathrm{i}} / \mathrm{k} \Omega \mathrm{cm}^{2}$ & $Q_{\mathrm{dl}} / \mu \mathrm{S} \mathrm{s}^{\mathrm{n}} \mathrm{cm}^{-2}$ & $n_{\mathrm{dl}}$ & $R_{\mathrm{ct}} / \mathrm{k} \Omega \mathrm{cm}^{2}$ \\
\hline CuNi & $58.95(0.78)$ & $0.86(0.01)$ & $6.06(5.14)$ & $66.72(30.09)$ & $0.52(0.03)$ & $17.88(11.20)$ \\
$\mathrm{T} 40$ & $16.83(5.46)$ & $0.80(0.04)$ & $1.36(0.34)$ & $23.15(10.80)$ & $0.65(0.09)$ & $63.49(6.48)$ \\
$\mathrm{T} 25 / \mathrm{t} 60$ & $13.21(4.14)$ & $0.80(0.08)$ & $6.11(5.58)$ & $9.57(3.87)$ & $0.66(0.14)$ & $84.09(15.29)$ \\
$\mathrm{t} 30 / 5 \mathrm{x}$ & $13.01(6.54)$ & $0.85(0.01)$ & $23.58(14.16)$ & $24.33(12.46)$ & $0.52(0.03)$ & $94.86(34.46)$ \\
$\mathrm{t} 10$ & $17.46(2.73)$ & $0.76(0.05)$ & $50.00(39.33)$ & $358.40(238.52)$ & $0.83(0.12)$ & $36.09(14.44)$ \\
2x & $19.68(2.49)$ & $0.85(0.01)$ & $14.11(7.05)$ & $19.40(1.72)$ & $0.50(0)$ & $73.27(15.43)$ \\
10x & $14.76(0.93)$ & $0.84(0.02)$ & $18.50(16.34)$ & $27.08(8.61)$ & $0.50(0)$ & $61.46(21.88)$ \\
\hline
\end{tabular}

For most of the samples, however, both resistance values $\left(R_{\mathrm{f}, \mathrm{i}}\right.$ and $\left.R_{\mathrm{ct}}\right)$ were still higher than those obtained for the blank sample, thus indicating that SA films provided corrosion protection even after two weeks of exposure to corrosive medium. The most significant decrease of $R_{\mathrm{f}, \mathrm{i}}$ and $R_{\mathrm{ct}}$ values was observed for 10x sample that exhibited the highest resistive values for the first day of exposure $3 \%$ $\mathrm{NaCl}$ (Table 5). The possible explanation for such behaviour would be that by increase of the film thickness, the number of defects in the film is also increased, especially in the outer layers, enabling thus their easier desorption from the surface. Obtained results confirm that the most protective SA film was formed on the sample $t 30 / 5 x$.

\section{Comparison of stearic and octadecylphosphonic acids films}

After examining the parameters of spray deposition of SA, ODPA films were prepared in the same way (t30/5x procedure), except at the final drying step that was conducted at $80{ }^{\circ} \mathrm{C}$ as applied in immersion deposition of ODPA [7]. Such prepared samples were immersed in $3 \% \mathrm{NaCl}$ solution and electrochemical measurements were conducted. The results of the polarization measurements are shown in Figure 6, and corrosion parameters obtained by Tafel extrapolation method from polarization curves are shown in Table 7.

(a)

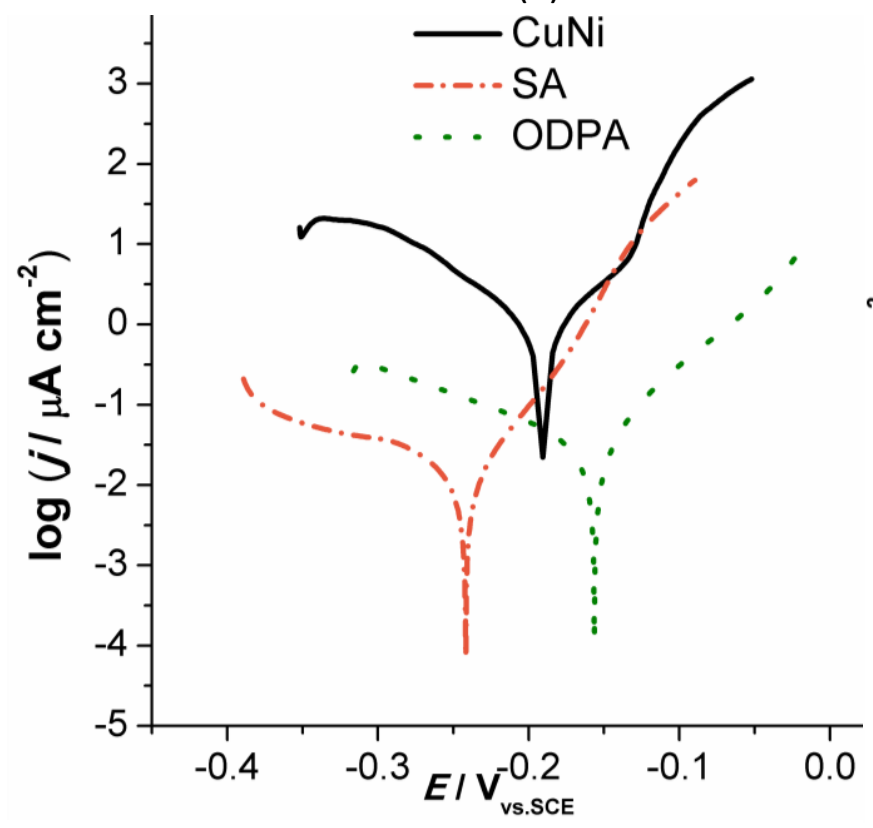

(b)

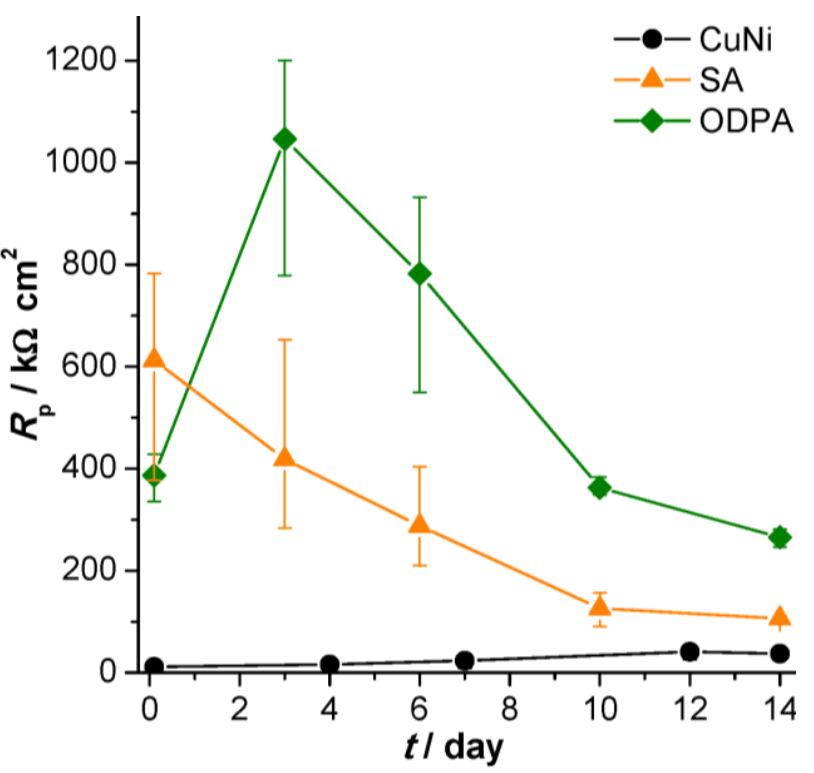

Figure 6. Comparison of equally prepared SA and ODPA films - results of polarization measurements: (a) polarization curves recorded after one hour immersion in $3 \% \mathrm{NaCl}$, and (b) polarization resistance as a function of immersion time for blank and treated samples. 
From polarization curves given in Figure $6 a$ it can be observed that while the protective films of SA significantly decreased cathodic current densities and only slightly anodic current densities, ODPA films reduced both anodic and cathodic current densities. Apart from acting as a barrier film, it may be assumed that ODPA is adsorbed on anodic sites of alloy thus preventing their dissolution. Protective efficiency of ODPA film was slightly lower than that of SA film. Also, on the $1^{\text {st }}$ day of exposure to $3 \% \mathrm{NaCl}$, SA film showed higher polarization resistance values compared to ODPA film. However, contrary to a continuous decrease in $R_{\mathrm{p}}$ values of SA films in time, $R_{\mathrm{p}}$ values of ODPA films, increased on the third day of immersion, probably due to the reorganization of molecules in the film into the more stable configuration. Later on, $R_{\mathrm{p}}$ values of ODPA samples decreased in time but remained always higher than those obtained for SA samples.

Studies were also conducted by EIS in order to better understand corrosion behaviour of ODPA samples (Figure 7).

Table 7. Corrosion parameters obtained for sample with ODPA protective layer. Values in parenthesis represent standard deviations

\begin{tabular}{cccccc}
\hline Sample & $E_{\text {corr }} / \mathrm{mV}$ vs. SCE & $j_{\text {corr }} / \mu \mathrm{A} \mathrm{cm}{ }^{-2}$ & $b_{\mathrm{a}} / \mathrm{mV} \mathrm{dec}^{-1}$ & $\left.-b_{\mathrm{c}} / \mathrm{mV} \mathrm{dec}^{-1}\right)$ & $\left.\eta_{\mathrm{i}} / \%\right)$ \\
\hline ODPA & $-185(28)$ & $0.11(0.07)$ & $43(4)$ & $69(3)$ & $90(6)$ \\
\hline
\end{tabular}

(a)

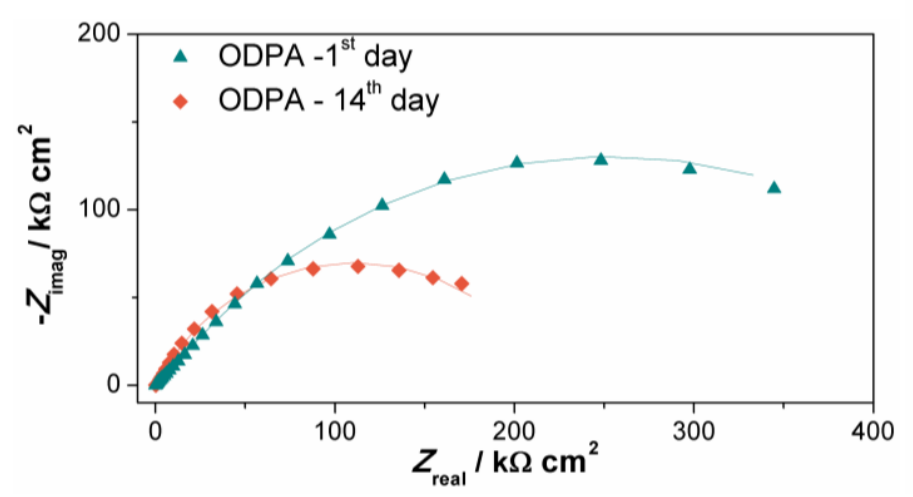

(b)

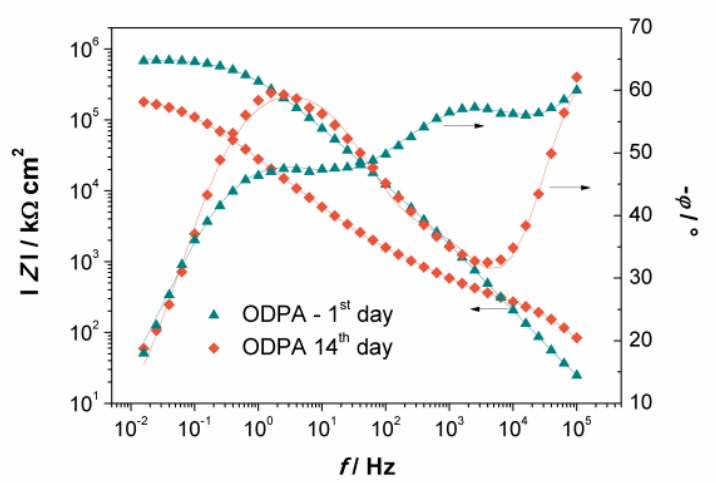

Figure 7. Nyquist (a) and Bode (b) impedance spectra for ODPA treated samples for the $1^{\text {st }}$ and $14^{\text {th }}$ day of immersion in $3 \% \mathrm{NaCl}$ solution.

EIS spectra given in Figure 7 confirm findings from polarization measurements that ODPA films initially provided lower corrosion protection than SA films (Figure 4), but this protection was much more stable in time. The shapes of phase angle - log frequency curves of Bode plots in Figure $7 \mathrm{~b}$ reveal that it was necessary to use the electrical equivalent circuit with three time constants to adequately fit EIS spectra measured for both the first and the last day of exposure to $3 \% \mathrm{NaCl}$. For this purpose, the electrical equivalent circuit given in Figure $5 b$ was used and the obtained impedance parameter values are presented in Table 8 (except for $R_{\mathrm{f}, \mathrm{o}}-Q_{\mathrm{f}, \mathrm{o}}$ elements).

Table 8. Impedance parameters for ODPA treated samples

\begin{tabular}{|c|c|c|c|c|c|c|}
\hline & $Q_{\mathrm{f}, \mathrm{i}} / \mu S \mathrm{~s}^{\mathrm{n}} \mathrm{cm}^{-2}$ & $n_{\mathrm{f}, \mathrm{i}}$ & $R_{\mathrm{f}, \mathrm{i}} / \mathrm{k} \Omega \mathrm{cm}^{2}$ & $Q_{\mathrm{dl}} / \mu S \mathrm{~s}^{\mathrm{n}} \mathrm{cm}^{-2}$ & $n_{\mathrm{dl}}$ & $R_{\mathrm{ct}} /$ \\
\hline & & & 47.97 & & & \\
\hline ODPA $-14^{\text {th }}$ day & $3.58(1.92)$ & $0.71(0.04)$ & $1.66(0.75)$ & $4.81(0.78)$ & $0.69(0.04)$ & $212.37(6.86)$ \\
\hline
\end{tabular}

Therefore, it appears that ODPA films were initially characterized by the presence of porous outer layers which might explain the lower protection exhibited by ODPA than SA films. However, by examining the impedance parameter values for the $14^{\text {th }}$ day of exposure to $3 \% \mathrm{NaCl}$, it is clear that 
$R_{\mathrm{ct}}$ value for ODPA sample was twice higher than for SA sample, while $Q_{\mathrm{dl}}$ values of ODPA sample were five times lower than for SA sample. Taking into account that polarization measurements (Figure 6a) showed that unlike SA, ODPA exhibited strong anodic current inhibition, and also that phosphonic acids are stronger than carboxylic acids, it may be proposed that ODPA molecules were more strongly bounded to CuNi surface than SA molecules. Thus, despite the formation of more porous surface layer, dissolution of strongly bounded ODPA molecules was more difficult than dissolution of SA molecules.

Results obtained in this work are similar to results obtained in our previous study [7], where ODPA films were prepared by dip-coating method, except for the fact that for dip-coating method, presence of porous outer layer was not clearly observed for the first day of exposure to $3 \% \mathrm{NaCl}$. However, the $R_{\mathrm{p}}$ values observed in this work for ODPA films formed by spraying are only slightly higher than those observed for ODPA films formed by dip-coating [7]. When comparing the results obtained for SA films prepared by dip-coating $[14,15]$ and spraying method, it appeared that films obtained by spraying initially exhibited higher corrosion protection than films obtained by dipcoating method. However, the resistance of sprayed samples decreased more significantly in time, eventually reaching the values obtained for dip-coated samples. Therefore, for preparation of protective SA and ODPA films, spraying method can be used instead of dip-coating method.

The thicknesses of obtained coatings can be roughly estimated from the film capacitance $\left(C_{f}\right)$ values as $C_{\mathrm{f}}$ value would be reversely proportional to the film thickness. According to Brug et al. [31], the pure capacitance $(C)$ value can be calculated using the relationship $C=\left(\left.Q R_{\mathrm{e}}\right|^{1-n}\right)^{1 / n}$. For SA film obtained by optimal preparation procedure $(5 x)$, the calculated $C_{f}$ was about $0.13 \mu \mathrm{F} \mathrm{cm}^{-2}$. In our previous work [15], the thickness of SA film obtained by dip-coating was determined by ellipsometry. It was found that SA film exhibiting $C_{\mathrm{f}}$ value of $0.88 \mu \mathrm{F} \mathrm{cm} \mathrm{cm}^{-2}$ has the thickness of about $18 \mathrm{~nm}$. Thus, one can estimate that SA film-5x has the thickness of approximate $120 \mathrm{~nm}$. In addition, it has to be taken into account that $C_{\mathrm{f}}$ value doesn't depend solely on the film thickness but also on dielectric constant of the film, which significantly depends on the amount of the water in film (film porosity). Anyway, it appears that films formed by spraying method were thicker than those formed by dip-coating method. This could explain better initial corrosion protection offered by spray deposited SA films. For ODPA film, inner and outer films were observed with $Q_{\mathrm{f}, \mathrm{o}}$ much lower than $Q_{\mathrm{f}, \mathrm{i}}$. However, the values of $n_{\mathrm{f}, \mathrm{i}}$ and $n_{\mathrm{f}, \mathrm{o}}$ were found lower than 0.75 which is considered as a threshold limit for the use of Brug equation and $C_{f}$ calculation.

\section{Surface studies}

FTIR spectroscopy was used to confirm stearic and phosphonic acid film formation and to analyse the alkyl chain ordering in the films. Characteristics of the methylene $\left(\mathrm{CH}_{2}\right)$ stretching region of the FTIR spectrum indicate whether or not an ordered film was present on the surface. A well-ordered film has been characterized as having alkyl chains in all trans configuration on the substrate with characteristic peaks at $v_{\mathrm{CH}_{2} \text { asymm }} \leq 2918 \mathrm{~cm}^{-1}$ and $v_{\mathrm{CH}_{25 y m m}} \leq 2850 \mathrm{~cm}^{-1}$. Shifts of these peaks to higher wavenumbers indicate a disordered film with gauche interactions in the alkyl chain [26-28]. Although, FTIR has been mainly used to characterize monolayer films, it was also applied in characterization of self-assembled and Langmuir-Blodgett multilayer films [29,30]. FTIR spectra presented in Figure 8 exhibit adsorption peaks characteristic for well-ordered films for both studied samples. Since the layers obtained in our study where not monolayers but multilayers, FTIR spectra cannot be used for accurate determination of ODPA bonding to CuNi surface as vibrations of upper layers dominate in the obtained spectra. 

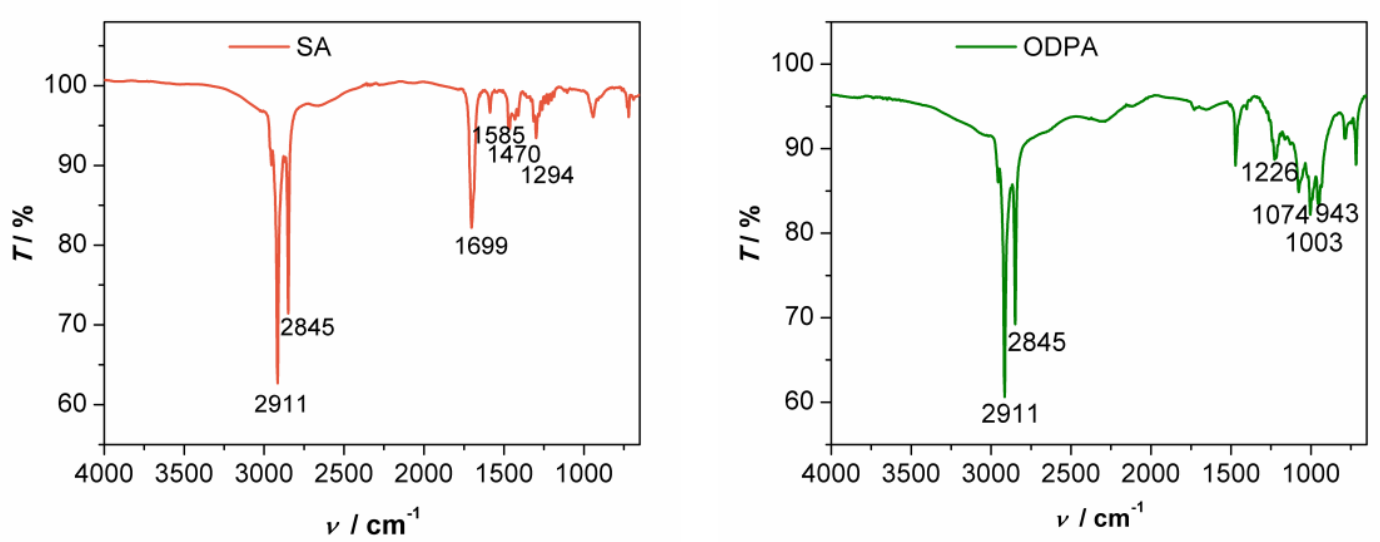

Figure 8. FTIR spectra of SA and ODPA layers deposited on CUNi alloy by selected spraying method

The water contact angle (CA) is commonly used to determine the wettability of modified surface but is also considered as a measure of the layer crystallinity. Well-ordered and densely packed SAMs $[11,26,30]$ have shown the water contact angle of around $108^{\circ}$. The water contact angles determined on SA and ODPA treated samples (Figure 9) showed such CA values indicating that the alkyl tail group was oriented towards environment.

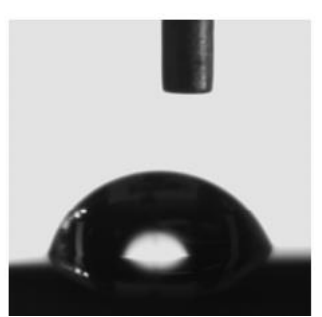

$(62 \pm 3)^{\circ}$

CuNi

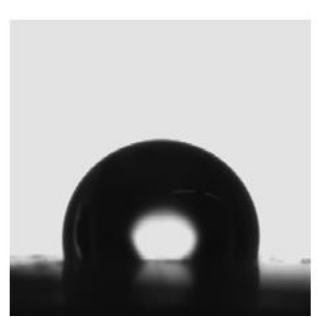

$(107 \pm 2)^{\circ}$

SA

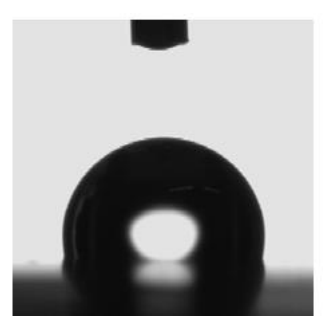

$(108 \pm 1)^{\circ}$

ODPA

Figure 9. Comparison of water contact angles on non-treated CUNi alloy and samples treated with SA and ODPA by selected spraying method

Freshly prepared SA and ODPA samples were examined by means of scanning electron microscopy, afterwards samples were exposed to $3 \% \mathrm{NaCl}$ solution for 14 days and examined by SEM again (Figure 10).
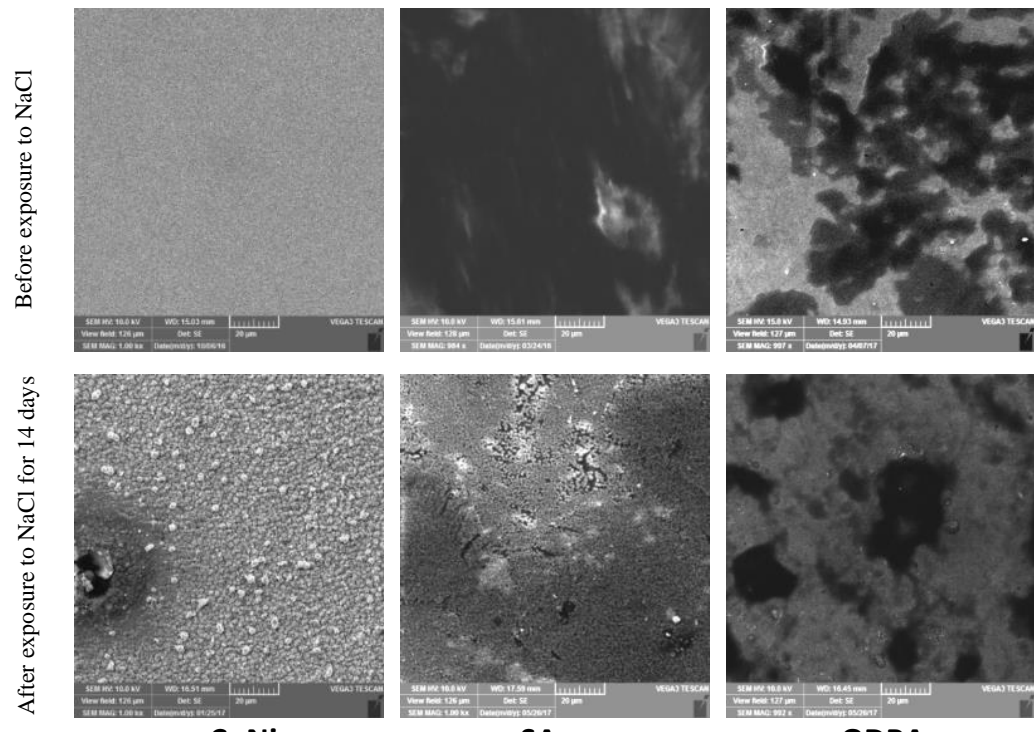

CuNi

SA

ODPA

Figure 10. SEM images of non-treated CUNi alloy and equally prepared SA and ODPA films before and after exposure to $3 \% \mathrm{NaCl}$ solution for 14 days 
Comparing the morphology of stearic and octadecylphosphonic acid films, it can be seen that after preparation of the sample, the stearic acid films appeared more uniform. However, after 14 days of exposure to corrosive media, SA films exhibited large number of cracks in the film, while ODPA films visually didn't change as much.

\section{Conclusion}

Studies conducted in this work confirmed that spraying method can be used to prepare protective films of SA and ODPA on CuNi alloy and that adequately prepared films exhibit similar properties to those observed for films formed by dip-coating method. Several parameters of spraying deposition method were examined, among which time elapsed between two sprays and number of sprays have the strongest influence on the film stability and its protective properties. Comparison of similarly prepared SA and ODPA films showed that initially, SA films provide better corrosion protection than ODPA films. Prolonged exposure to corrosive medium, however, showed faster decline of SA film protective properties than that of ODPA film, what is related to stronger attachment of phosphonic acids on CuNi surface. It was also shown that spray formed protective films provide corrosion protection to the underlying CuNi alloy even after 14 days of continuous exposure to corrosive medium.

Acknowledgements: The research leading to these results has received funding from Croatian Science Foundation under grant agreement 9.01/253.

\section{References}

[1] C. Queffelec, M. Petit, P. Janvier, D. A. Knight, B. Bujoli, Chemical Reviews 112 (2012) 3777 - 3807.

[2] P. J. Hotchkiss, S. C. Jones, S. A. Paniagua, A. Sharma, B. Kippelen, N. R. Armstrong, S. R. Marder, Accounts of Chemical Research 45 (2012) $337-346$.

[3] R. Michel, J.W. Lussi, G. Csucs, I. Reviakine, G. Danuser, B. Ketterer, J. A. Hubbell, M. Textor, N. D. Spencer, Langmuir 18 (2002) $3281-3287$.

[4] S. Flink, F. C. J. M. van Veggel, D. N. Reinhoudt, Advanced Materials 12 (2000) 1315-1328.

[5] S. P. Pujari, L. Scheres, A. T. M. Marcelis, H. Zuilhof, Angewandte Chemie International Edition 53 (2014) 6322-6356.

[6] S. Ghareba, S. Kwan, S. Omanovic, Journal of Electrochemical Science and Engineering 5 (2015) 157172.

[7] E. Kristan Mioč, Z. Hajdari Gretić, H. Otmačić Ćurković, Corrosion Science 134 (2018) 189-198.

[8] A. Ulman, Chemical Reviews 96 (1996) 1533-1554.

[9] G. M. Whitesides, G. S. Ferguson, D. Allara, D. Scherson, L. Speaker, A. Ulman, Critical Reviews in Surface Chemistry 3 (1993) 49-65.

[10] T. Ishizaki, M. Okido, Y. Masuda, N. Saito, M. Sakamoto, Langmuir 27 (2011) 6009-6017.

[11] R. Quiñones, A. Raman, E. S. Gawalt, Thin Solid Films 516 (2008) 8774-8781.

[12] J. Telegdi, H. Otmačić Ćurković, K. Marušić, F. Al-Taher, E. Stupnišek-Lisac, E. Kálmán Chemical and Biochemical Engineering Quarterly 21 (2007) 77-82.

[13] I. Milošev, M. Metikoš-Huković, Ž. Petrović, Materials Science and Engineering C 32 (2012) 2604-2616.

[14] Z. Hajdari, H. Otmačić Ćurković, V. Čadež, S. Šegota, Journal of the Electrochemical Society 163 (2015) C145-C155.

[15] Z. Hajdari Gretić, E. Kristan Mioč, V. Čadež, S. Šegota, H. Otmačić Ćurković, S. Hosseinpour, Journal of the Electrochemical Society 163 (2016) C937-C944.

[16] G. A. Buckholt, E. S. Gawalt, Materials 5 (2012) 1206-1218.

[17] A. Bulusu, S. A. Paniagua, B. A. MacLeod, A. K. Sigdel, J. J. Berry, D. C. Olson, S. R. Marder, S. Graham, Langmuir 29 (2013) 3935-3942.

[18] D. K. Schwartz, Annual Review of Physical Chemistry 52 (2001) 1017-137.

[19] S. G. Brass, G. Ehrlich, Physical Review Letters 57 (1986) 2532-2535. 
[20] H. Zang, X. Wang, L. Zheng, X. Jiang, International Journal of Heat and Mass Transfer, 44 (2001) 45794592.

[21] D. H. Charlesworth W. R. Marshall, AIChE Journal 6 (1960) 9-23.

[22] Z. Grubač, I. Škugor Rončević, M. Metikoš-Huković, R. Babić, M. Petravić, R. Peter, Journal of the Electrochemical Society 159 (2012) C253-C258.

[23] P. Wang, C. Liang, B. Wu, N. Huang, J. Li, Electrochimica Acta 55 (2010) 878-883.

[24] B. R. A. Neves, M. E. Salmon, P. E. Russell, E. B. Troughton, Langmuir, 17 (2001) 8193-8198.

[25] J. R. Macdonald, Impedance Spectroscopy, Emphasizing Solid Materials and Systems, John Wiley \& Sons, Inc., New York, USA, 1987.

[26] A. Raman, M. Dubey, I. Gouzman, E.S. Gawalt, Langmuir 22 (2006) 6469-6472.

[27] D.L. Allara, R.G. Nuzzo, Langmuir 1 (1985) 45-52.

[28] A. Raman, R. Quiñones, L. Barriger, R. Eastman, A. Parsi, E.S. Gawalt, Langmuir 26 (2010) 1747-1754.

[29] F. Kimura, J. Umemura, T. Takenaka, Langmuir 2 (1986) 96-101.

[30] A. Baptiste, A. Gibaud, J.F. Bardeau, K. Wen, R. Maoz, J. Sagiv, B. M. Ocko, Langmuir 18 (2002) 39163922.

[31] G. J. Brug, A. L. G. van der Eeden,M. Sluyters-Rehbach, J. H. Sluyters, Journal of Electroanalytical Chemistry 176 (1984) 275-295.

(C)2020 by the authors; licensee IAPC, Zagreb, Croatia. This article is an open-access article distributed under the terms and conditions of the Creative Commons Attribution license (http://creativecommons. org/licenses/by/4.0/) 